\section{Precision-made appliances for snoring and sleep apnoea}

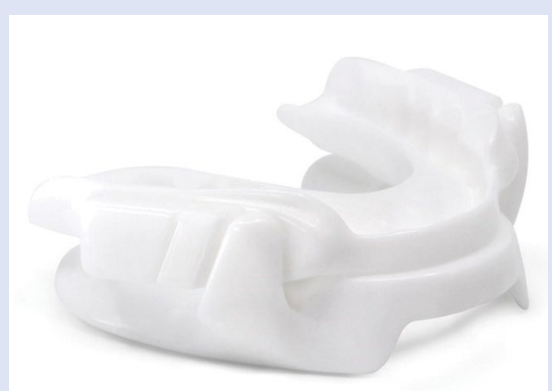

Dentists now have the opportunity to prescribe world-leading precision-made oral appliances from Panthera Dental (Quebec, Canada) for the treatment of obstructive sleep apnoea (OSA) and snoring, which affects billions of people worldwide and is a growing market. The launch in UK and Ireland follows other new market launches this year in France and Germany and the opening of a new office in Paris.

Panthera Dental's custom-made Digital Sleep Apnoea Devices (D-SADs) are designed and manufactured using highprecision proprietary CAD/CAM processes and $100 \%$ medical grade type- 12 polyamide nylon. This material has proved to be a game changer in oral appliance manufacture,

being at once light, smooth, strong, flexible, highly durable and resistant to bruxism. The D-SADs are also extremely customisable with a wide range of plateaus and bands allowing for over 300 design combinations. All Panthera appliances can be adapted to every possible patient morphology, from basic to the most complex. They are uniquely simple and easy to care for.

Thousands of dentists in UK and Ireland have already recognised the opportunity to play a pivotal role in the identification and treatment of OSA. Through training provided by appliance manufacturers or national societies like the British Society of Dental Sleep Medicine (BSDSM), they can quickly become qualified to offer Oral Appliance Therapy (OAT) in their practices.
Indeed, Panthera is organising expert-led training days in the UK and Ireland in November to teach dentists the essentials of dental sleep medicine.

Dentists are well placed to identify those with problem snoring and OSA. They can easily start the conversation with a simple question like 'do you snore?' and then screen their patients using questionnaires.

Panthera Dental's 'Industry 4.0' manufacturing process uses robotics and AI to produce appliances with unmatched precision and traceability, enabling dentists around the world to help many suffering patients live longer, better and healthier lives and provide an alternative solution to CPAP therapy. The Panthera D-SADs are indicated for snoring and OSA, have FDA $510(\mathrm{k})$ clearance and are CE-marked.

More information about Panthera Dental is available at www.pantheradental.com.

More information about D-SADs is available at www.pantherasleep.com.

\title{
Faster scanning and improved visualisation
}

Align Technology, Inc. ('Align'), the leading global medical device company that designs, manufactures and sells the Invisalign system of clear aligners, iTero intraoral scanners and exocad CAD/ CAM software for digital orthodontics and restorative dentistry, has announced the new iTero Workflow 2.0 software release with advanced features that provide enhanced intraoral image sharpness for clearer hard and soft tissue details to aid in treatment diagnosis, while also driving practice efficiency, patient engagement, and a more seamless end-to-end digital treatment experience for doctors and their patients.

The newly released iTero Workflow 2.0 software features include:

- Faster scanning: Enables a faster and smoother all-in-one scan for maximum efficiency with $20 \%$ less waiting for processing time on the iTero Element Plus Series scanners and 50\% faster movement and $25 \%$ faster rotation during scanning for efficient daily use and ease of learning on all iTero Element scanners

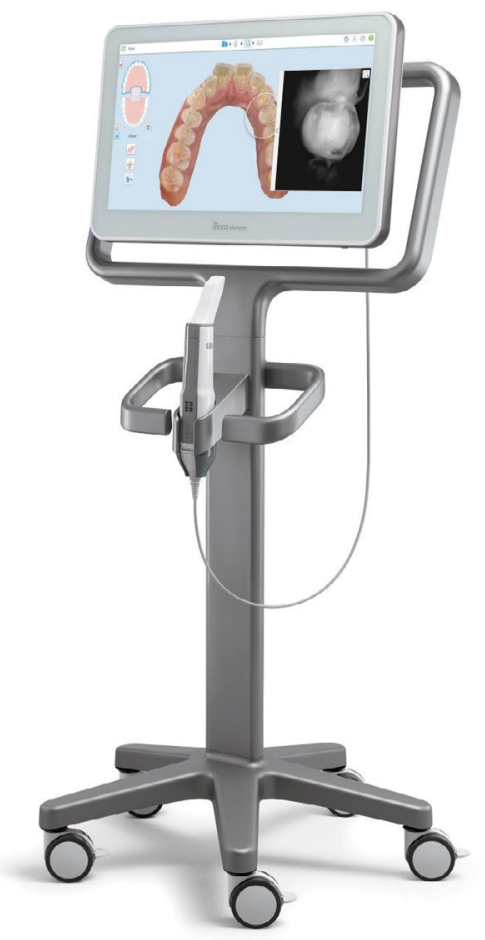

- Improved visualisation: The integrated 3D intraoral camera included in the iTero Element 5D Plus imaging system provides enhanced sharpness and improved image quality powered by advanced AI capabilities to deliver clearer soft and hard tissue details to support diagnosis. In addition, the enhanced capabilities allow clinics to efficiently use intraoral scan images in place of traditional intraoral photos as they can capture multiple intraoral images at different angles automatically with one scan

- Next-level patient communication tools: The new Snapshot tool and iTero Scan Report provide dentists and their staff with the ability to capture information such as Invisalign Outcome Simulator projections and share it digitally with their patients, allowing patients to make more confident decisions - in the dental chair or at home - which may lead to higher treatment acceptance.

iTero Workflow 2.0 software features are being rolled out regionally and are expected to be available in all markets where the iTero Element Plus imaging systems are available. Discover more at https://bit.ly/2ROw0fD. 